\title{
A high order off-lattice kinetic method for high speed flows with a moderate Knudsen number
}

\author{
Peng Wang ${ }^{1}$ and Yonghao Zhang ${ }^{1, a)}$ \\ ${ }^{1}$ James Weir Fluids Laboratory, Department of Mechanical and Aerospace Engineering, University of Strathclyde, \\ Glasgow G1 1XJ, UK \\ ${ }^{a)}$ Corresponding author: yonghao.zhang@strath.ac.uk
}

\begin{abstract}
In this work, in the framework of the discrete unified gas kinetic scheme, a high order off-lattice kinetic method is proposed for high speed rarefied gas flows. The velocity distribution function is expanded on the basis of the Hermite polynomial to a certain order to ensure the conservation of the corresponding moments. The sod shock tube problem and two dimensional liddriven cavity flow in the hydrodynamic and early transition regimes are performed to assess the proposed method. In particular, the performance of third and fourth order of truncated Hermite polynomial associated with several on and off-lattice discrete velocity sets are evaluated. It is confirmed from the simulation results that, with the fourth order Hermite polynomial expansion the proposed method can accurately reproduce the thermal and fully compressible hydrodynamic flow. However, it is surprisingly found that the performance of D2Q16 is better than that of D2Q17, and is even better than those of D2Q37 and D2Q25 for flows in the early transition regime, suggesting that the even number of velocity points without the zero velocity seems to have a better capacity than the odd one in capturing rarefaction effect. This indicates that the molecular velocity space discretized without the zero velocity may be a promising way to reduce the number of velocity point.
\end{abstract}

\section{Introduction}

The Boltzmann equation based kinetic methods have undergone an impressive growth in the past two decades, both in their theoretical foundations and in their range of applications in many areas of fluid mechanics. In gas kinetic theory, the gas dynamics of molecules are governed by a velocity distribution function $f$, and through its ChapmanEnskog expansion to up to zero and first orders, the hydrodynamic equations, e.g., the Euler and Navier-Stokes-Fourier equations, can be recovered, respectively [1].

There are two main classes of kinetic methods depending on their scope of application. The concentration of the first class is to the continuum flow, including the lattice Boltzmann method [2] (LBM) and gas kinetic method (GKS) [3]. Compared with the GKS with continuous molecular velocity space for compressible flows, the LBM, with strong coupling of the discrete molecular velocity and spatial spaces, is specially designed for the nearly incompressible flows. The LBM is not only much easier to be implemented, but also is less dissipative than the GKS due to the explicit deduction of the numerical viscosity in LBM. Consequently, the LBM has been extensively applied to many fields, in particular to the multiphase flow and the porous media flow. Another kind of the kinetic method is usually called the discrete velocity method (DVM) that approximates the continuous molecular velocity space by discrete velocity points, so that the resulting equations can be solved numerically [4]. In the traditional DVM, the Boltzmann model equation is explicitly solved through the operator splitting method, where the time step and cell size are limited by the mean collision time and mean free path of gas molecules, respectively. Therefore, the DVM works well for highly rarefied gas flows, but encounters great difficulties for near-continuum and continuum flows [5].

In fact, according the definition, the LBM can be viewed as a special DVM. However, the special particle transport and collision mechanism requires the coupling discrete molecular velocities and spatial grids, which limits the LBM just working for the incompressible continuum flows. In order to validity range of the LBM, several types of the high order LBM [6, 7, 8, 9, 10] have also been proposed for the compressible flows on the basis of Grad's work [11], which builds a systematic link between the kinetic theory and fluid mechanics. Typically, a fourth order approximation is mandatory for thermal and fully compressible flow [7]. However, with the higher order approximation of the

31st International Symposium on Rarefied Gas Dynamics

AIP Conf. Proc. 2132, 130013-1-130013-7; https://doi.org/10.1063/1.5119633

Published by AIP Publishing. 978-0-7354-1874-5/\$30.00

130013-1 
distribution function, more discrete molecular velocity points are required to accurately compute the moments. As a result, on-lattice discrete velocity stencil is also broader, which not only actually induces much more trouble in implementing the boundary condition, but makes the scheme extremely unstable. Therefore, some kinds of technique are coupled to improve the numerical stability of the high order LBM $[12,7]$. However, these ways make the LBM lost its simplicity nature.

We note that all defects of the high order LBM origin from the on-lattice streaming mechanism, while the traditional DVM, which uses off-lattice discrete velocity sets, only works well in the transitional and free molecular regimes, and is computational costly for the continuum flows in which the mean free path of molecules is far smaller than the mesh size. Recently, discrete unified gas kinetic scheme (DUGKS) has been developed for flows covering all the regimes $[13,14]$, which can perfectly remedy all the mentioned drawbacks in both the LBM and DVM. In this work, the high order Hermite polynomial expansion with the proper discrete velocity sets are implemented in the DUGKS. The order of Hermite expansion and several types of the discrete velocity sets are assessed in detail by performing the one dimensional shock tube test and two dimensional lid-driven cavity flow.

\section{High order discrete unified gas kinetic scheme}

In this work, the high order DUGKS is based on the Bhatnagar-Gross-Krook (BGK) [15], which takes the form of

$$
\frac{\partial f}{\partial t}+\xi \cdot \nabla f=-\frac{1}{\tau}\left[f-f^{e q}\right]
$$

where $f(\boldsymbol{x}, \boldsymbol{\xi}, t)$ is the velocity distribution function (VDF) of gas molecules at the position $\boldsymbol{x}=(x, y, z)$ and the time $t$, with $\boldsymbol{\xi}=\left(\xi_{x}, \xi_{y}, \xi_{z}\right)$ being the molecular velocity. The collision time $\tau$ in Eq. (1) is evaluated from the viscosity $\mu$ and the pressure $p$ by $\tau=\mu / p$. The Maxwellian equilibrium distribution function $f^{e q}$ is given as

$$
f^{e q}=\frac{\rho}{(2 \pi R T)^{3 / 2}} \exp \left(-\frac{c^{2}}{2 R T}\right)
$$

where $\boldsymbol{c}=\boldsymbol{\xi}-\boldsymbol{U}$ is the peculiar velocity and $\boldsymbol{U}=\left(U_{x}, U_{y}, U_{z}\right)$ is the macroscopic flow velocity. The conservative variables $\boldsymbol{W} \equiv(\rho, \rho \boldsymbol{U}, \rho E)^{T}$ are calculated from the velocity moments of the velocity distribution function, $\boldsymbol{W}=$ $\int \boldsymbol{\psi} f \mathbf{d} \xi$, where $\boldsymbol{\psi}=\left(1, \boldsymbol{\xi}, \frac{1}{2} \xi^{2}\right)^{T}$. Note that for ideal gas the temperature is related to the total energy as $\rho E=\frac{1}{2} \rho U^{2}+$ $\frac{3}{2} \rho R T$, with $R$ is the Boltzmann constant.

On the basis of Grad's work [11], the VDF in Eq. (1) can be approximated by its projection onto a Hilbert subspace spanned by the first $N$ polynomials without changing the first $N$ moments, i.e.

$$
f(\boldsymbol{x}, \boldsymbol{\xi}, t) \approx f^{N}(\boldsymbol{x}, \boldsymbol{\xi}, t)=\omega(\boldsymbol{\xi}) \sum_{n=0}^{N} \frac{1}{n !\left(R T_{0}\right)^{n}} \boldsymbol{a}^{n}(\boldsymbol{x}, t) \mathcal{H}^{n}(\boldsymbol{\xi}),
$$

where

$$
\boldsymbol{a}^{n}(\boldsymbol{x}, t)=\int f(\boldsymbol{x}, \boldsymbol{\xi}, t) \mathcal{H}^{n}(\boldsymbol{\xi}) d \boldsymbol{\xi}
$$

The details of $\omega, \boldsymbol{a}^{n}$, and $\mathcal{H}^{n}$ refer to literatures of Shan et al. $[6,16]$. It is well recognized that the third $(N=3)$ and fourth $(N=4)$ order Hermite polynomials could accurately reproduce the isothermal, and thermal and fully compressible flows [7], respectively. Therefore, the $N$-th moments of the VDF can be exactly represented by the discrete velocities, if the degree of precision of the quadrature is at least $2 N+1$.

For two-dimensional case, two compact on-lattice high order velocity sets, i.e. $\mathbf{D}_{2,7}^{17}$ and $\mathbf{D}_{2,9}^{37}$ (see Table 1), are usually applied in high order LBM [7, 16]. Based on the criteria presented by Shan et al. [6], the use of $\mathbf{D}_{2,7}^{17}$ and $\mathbf{D}_{2,9}^{37}$ for Gauss-Hermite quadrature, which has 7-th and 9-th degree of quadrature precisions, respectively, can accurately obtain the moments of the VDF up to $N=3$ and $N=4$, respectively. In addition, the off-lattice Gauss-Hermite discrete velocity sets of $\mathbf{D}_{2,7}^{16}$ and $\mathbf{D}_{2,9}^{25}$, which are generated by the tensor product of $\mathbf{D}_{1,7}^{4}$ and $\mathbf{D}_{1,9}^{5}$ (refer to Table 1 in [6]), respectively, are also evaluated. Therefore, the third and fourth order moments of the VDF can be accurately obtained by using the $\mathbf{D}_{2,7}^{16}$ and $\mathbf{D}_{2,9}^{25}$, respectively. In order words, the isothermal flow with a small variation of the temperature, and the thermal and fully compressible flow can be accurately represented by $\mathbf{D}_{2,7}^{17}$ and $\mathbf{D}_{2,7}^{16}$ with third 
TABLE 1. Summary of the high order two-dimensional on-lattice velocity sets. Here the convention $\mathbf{D}_{n, k}^{m}$ is adopted to summarize all the characteristic of interest, namely, the number of discrete velocities $n$, the quadrature order $k$, and the number of dimensions $m$. In addition, p represents the number of discrete speeds of each velocity group, while their associated weights compose the right part of the table.

\begin{tabular}{lcccc}
\hline Group & $\xi_{i}$ & $\mathrm{p}$ & $D_{2,7}^{17}$ & $D_{2,9}^{37}$ \\
\hline 1 & $(0,0)$ & 1 & $(575+193 \sqrt{193}) / 8100$ & 0.23315066913235250228650 \\
\hline 2 & $(1,0)$ & 4 & $(3355-91 \sqrt{193}) / 18000$ & 0.10730609154221900241246 \\
\hline 3 & $(1,1)$ & 4 & $(655+17 \sqrt{193}) / 27000$ & 0.05766785988879488203006 \\
\hline 4 & $(2,0)$ & 4 & & 0.01420821615845075026469 \\
\hline 5 & $(2,1)$ & 8 & & 0.00535304900051377523273 \\
\hline 6 & $(2,2)$ & 4 & $(685-49 \sqrt{193}) / 54000$ & 0.00101193759267357547541 \\
\hline 7 & $(3,0)$ & 4 & $(1445-101 \sqrt{193}) / 54000$ & 0.00024530102775771734547 \\
\hline 8 & $(3,1)$ & 8 & & 0.00028341425299419821740 \\
\hline $1 / \sqrt{R T_{0}}$ & & & $\sqrt{5(25+\sqrt{193}) / 72}$ & 1.19697977039307435897239 \\
\hline
\end{tabular}

order approximation, and $\mathbf{D}_{2,9}^{37}$ and $\mathbf{D}_{2,9}^{25}$ with fourth order approximation, respectively. For sake of clarity, in the following, $\mathbf{D}_{m, k}^{q}$ is denoted by $\mathbf{D} m \mathbf{Q} q$.

In order to evaluate the capacity of the high order Hermite polynomial expansion associated with the given discrete velocity sets, an appropriate numerical method, which can describe high speed flows in all regimes, should be used. Therefore the discrete unified gas-kinetic scheme [13,14] (DUGKS) for the Boltzmann model equation is employed in this work, which has been developed for flows in the whole regimes. In DUGKS, with the intrinsic coupling of molecular collision and transport processes in the determination of the flux across the cell interface, the computational time step and mesh size are not limited by the collision time and mean free path of gas molecules, respectively, so that the multiscale flow physics can be captured from the hydrodynamic to free molecular regimes. The capacity of DUGKS has been evaluated for gas flows in all flow regimes by comparing with the most reliable methods in the corresponding regimes [5, 17]. In addition, the DUGKS has a distinguish performance in term of the numerical stability. Most importantly, the DUGKS is an off-lattice method, which is convenient to assess any type of discrete velocities, including both on and off-lattice molecular velocity points. Please refer the references $[13,14]$ for detailed informations about the DUGKS. Note that in this work, although the high order DUGKS is based on the BGK model with the Prandtl number being unit, the other model equations such as the Shakhov model [18] and Ellipsoidal Statistical model [19] can be implemented straightforwardly.

\section{Results and discussion}

\section{Sod shock tube}

In order to evaluate accuracy of the high order DUGKS with different velocity sets for high speed flows in the near continuum regime, the case of Sod shock tube is firstly simulated. The computational domain is $0 \leq x \leq 1$, and initially the density, velocity, and pressure are set to be

$$
\left(\rho_{1}, u_{1}, p_{1}\right)=(1.0,0.0,1.0) \quad 0 \leq x \leq 0.5 ; \quad\left(\rho_{2}, u_{2}, p_{2}\right)=(0.125,0.0,0.1) \quad 0.5<x \leq 1 .
$$

The gas considered is modeled as hard sphere molecules such that the viscosity is determined as $\mu=\mu_{0}\left(T / T_{0}\right)^{0.5}$, where $\mu_{0}$ is the reference viscosity at reference temperature $T_{0}$. The reference mean-free-path $\lambda_{0}$ is then changed by adjusting the $\mu_{0}$,

$$
\lambda_{0}=\frac{\mu_{0}}{p_{0}} \sqrt{\frac{\pi R T_{0}}{2}}
$$


where $p_{0}=\rho_{0} R T_{0}$ is the reference pressure. Here we take $\rho_{1}, p_{1}$, and $T_{1}$ as the reference density, pressure, and temperature, respectively. With different $\mu_{0}$, the flow will have different degree of rarefaction, which can be used to test the capability of numerical method at various molecular velocity discretizations for flows in different regimes. The degree of gas rarefaction is defined by the Knudsen number $(K n)$ as $K n=\lambda / L$, where $L$ is the characteristic length. In this case, $L=1$ is the length of the test domain.

The reference results are obtained by approximating the molecular velocity space using 201 discrete velocities uniformly distributed in $\left[-10 \sqrt{2 R T_{1}}, 10 \sqrt{2 R T_{1}}\right]$, and the Newton-Cotes quadrature is used to evaluate the velocity moments. The CFL number is set to be 0.5 in all simulations, and the output time is $t=0.15$, which has been normalized by $L / \sqrt{2 R T_{1}}$. In the computation the uniform grid with 200 cells is used to cover the physical domain for all cases.
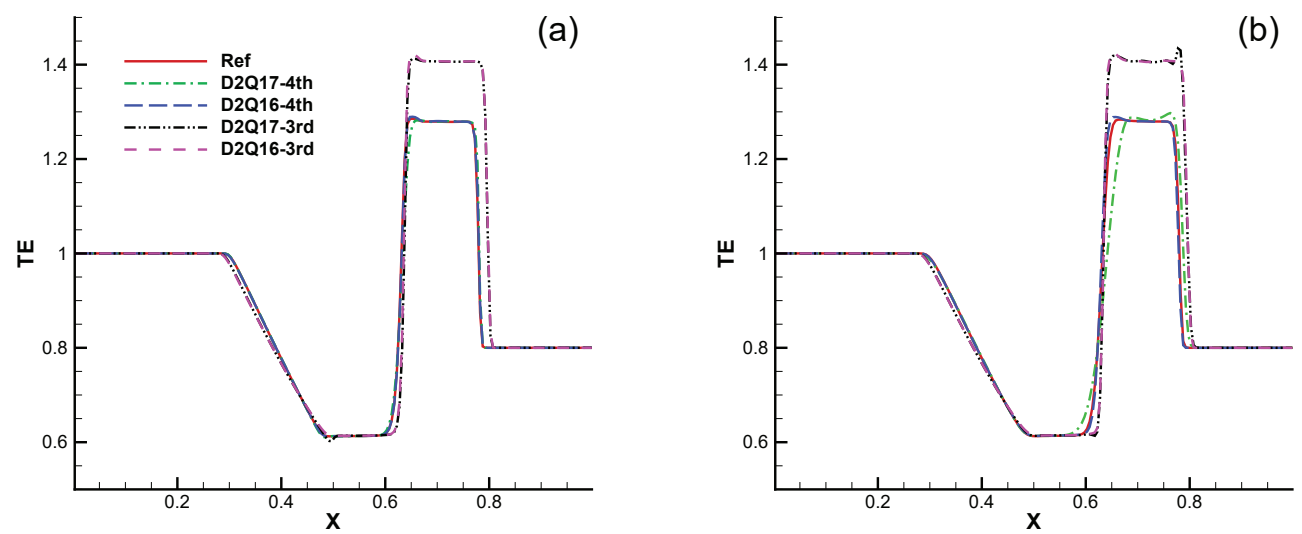

FIGURE 1. Temperature profiles of the shock tube test when $t=0.15$ : (a) $\mu_{0}=1.0 \times 10^{-5}$, and (b) $\mu_{0}=1.0 \times 10^{-4}$.
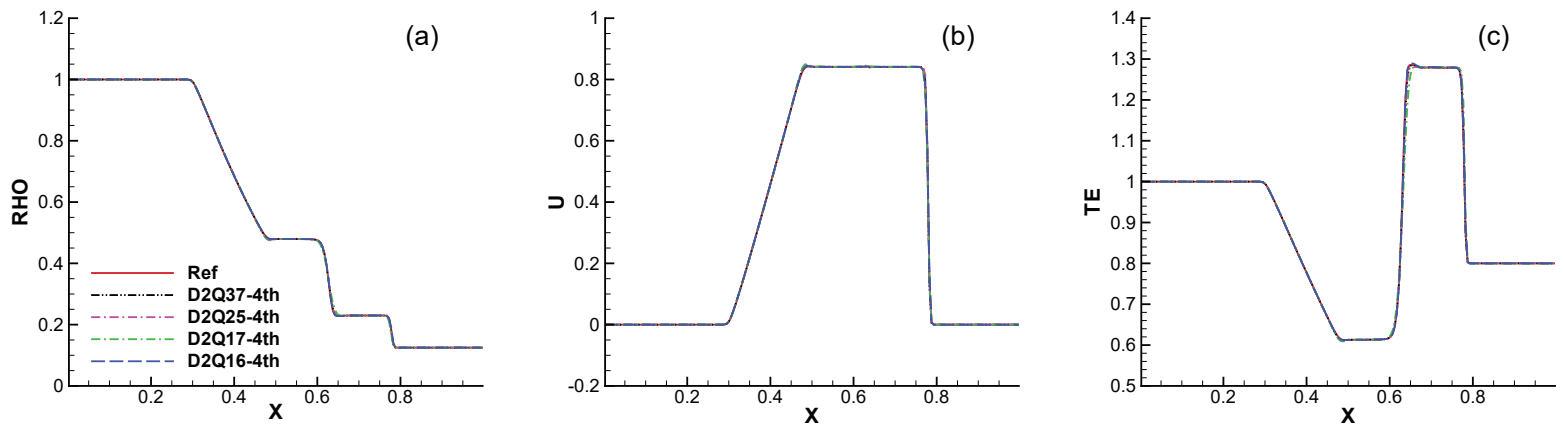

FIGURE 2. Density (a), velocity (b), and temperature (c) profiles of the shock tube test for $\mu_{0}=1.0 \times 10^{-5}$ with $t=0.15$.

The temperature profiles for $\mu_{0}=1.0 \times 10^{-5}$ and $1.0 \times 10^{-4}$ obtained from third and fourth order approximations with $\mathbf{D} 2 \mathbf{Q} 17$ and $\mathbf{D} 2 \mathbf{Q} 16$ are given in Fig. 1. In these cases the corresponding Knudsen numbers at the left boundary are about $K n=1.8 \times 10^{-5}$ and $1.8 \times 10^{-4}$, respectively, thereby the flows fall in the hydrodynamic regime. It is interestingly found that, although the heat flux cannot be conservatively obtained using the given velocity sets, the results from fourth order expansion is much better than those of the third one with which the temperature in downstream is overestimated by more than $10 \%$, due to the absent part of heat flux, as analyzed by the Chapman Enskog expansion in [7]. This suggests that the fourth order truncation of the Hermite polynomial is necessary to accurately predict the thermal dynamics. In addition, it is noticed that for the case of fourth order truncation of the VDF, the results from D2Q16 without zero molecular velocity, especially adjacent to the discontinuity in downstream, is much better 

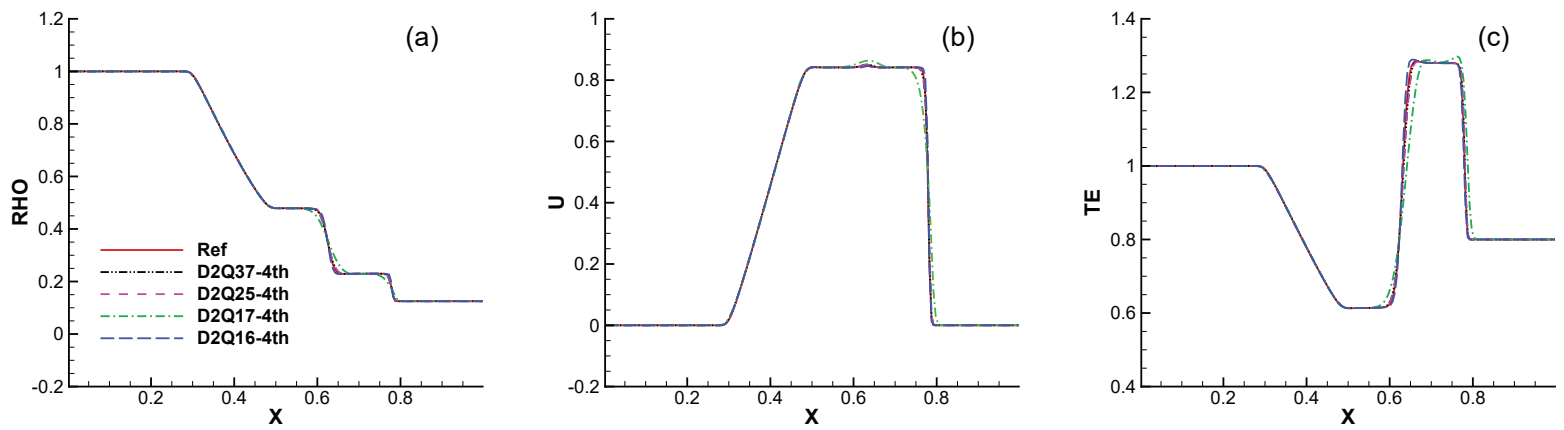

FIGURE 3. Density (a), velocity (b), and temperature (c) profiles of the shock tube test for $\mu_{0}=1.0 \times 10^{-4}\left(K n=1.8 \times 10^{-4}\right)$ with $t=0.15$.

than that of D2Q17 with the zero molecular velocity, when compared with the reference solutions. Therefore, in the following simulations only the fourth order approximation is considered for this case.

Figure 2 shows density, velocity, and temperature profiles for $\mu_{0}=1.0 \times 10^{-5}$ with fourth order expansion of the VDF associated with both on and off-lattice discrete velocity sets. Good agreements with the reference solutions are achieved, although with the D2Q16 and D2Q17 heat flux cannot be computed conservatively. When $\mu_{0}=1.0 \times 10^{-4}$, see Fig. 3, the results of DUGKS with fourth order approximation and various discrete velocity sets agree well with the reference solution, except that noticeable discrepancies are observed for D2Q17. Note that the result for larger reference viscosities are not shown here, but we can stress that due to the increasing rarefaction effect, the present discrete velocity sets already cannot show acceptable results.

\section{Lid-driven cavity flow}
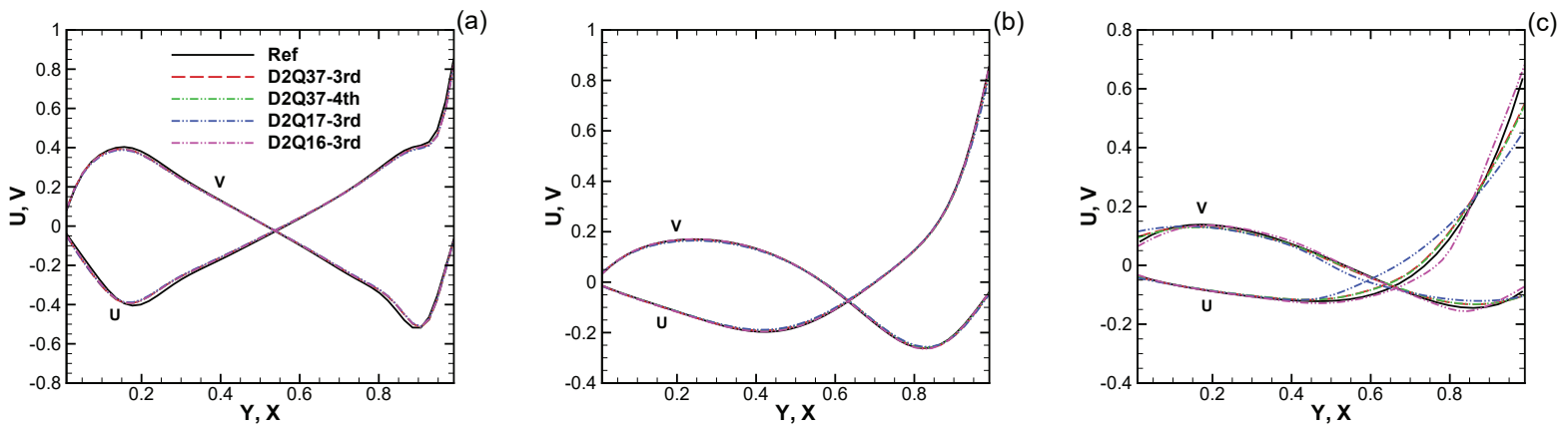

FIGURE 4. The horizontal and vertical velocity profiles along the vertical and horizontal centerlines of the cavity, respectively for $M a=1$ with $\mathrm{Kn}=0.001,0.01$, and 1 (from left to right column). The velocity is normalized by the lid-driven speed.

In order to further validate the capacity of high order Hermite polynomial expansion at different discrete velocity sets, two-dimensional lid-driven cavity flow for $K n=0.001,0.01$, and 0.1 with $M a=1$ are presented. Here the Mach number is defined by the lid driven velocity over the sound speed. In this case, the fully diffusive boundary condition is applied to the walls [14]. The results from D2Q37 with fourth order approximation, and D2Q17 and D2Q16 with third order one are compared with the reference solutions. The results obtained from D2Q37 with third order approximation are also given to show the effect of the order of Hermite polynomial expansion on accuracy. The reference solutions are obtained from the DUGKS with non-expansion equilibrium distribution function and sufficient discrete velocity points, see setup details in [5].

Bearing in mind that the third order truncation of the Hermite polynomial expansion can accurately recover the momentum in the framework of hydrodynamic flow, therefore, if the precision of the quadrature is sufficiently high, 

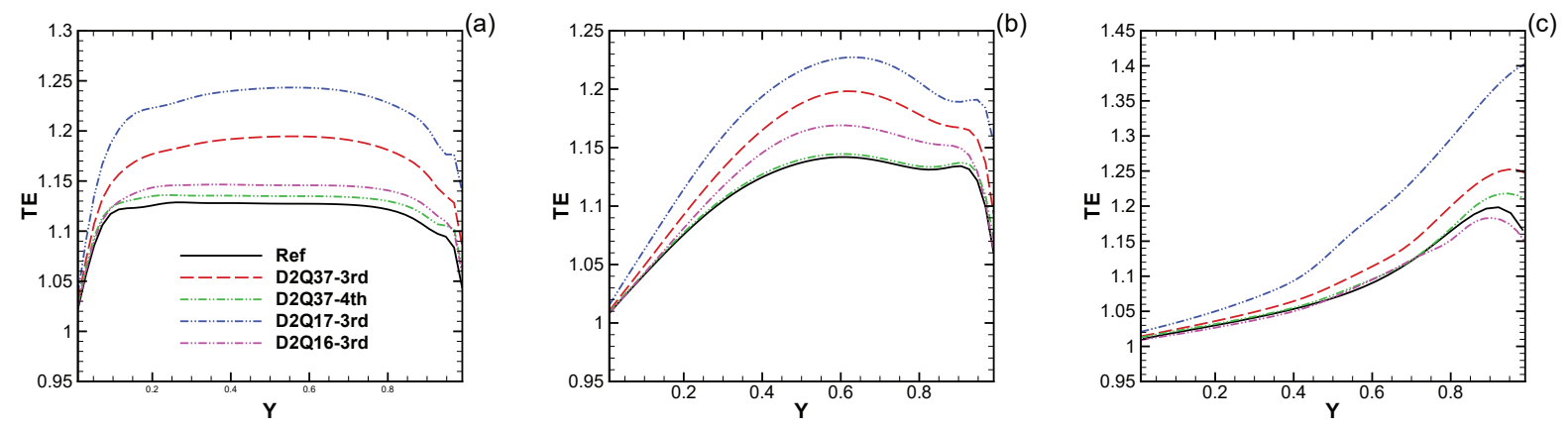

FIGURE 5. The temperature along the vertical centerline of the cavity for $M a=1$ with $\mathrm{Kn}=0.001,0.01$, and 1 (from left to right column).
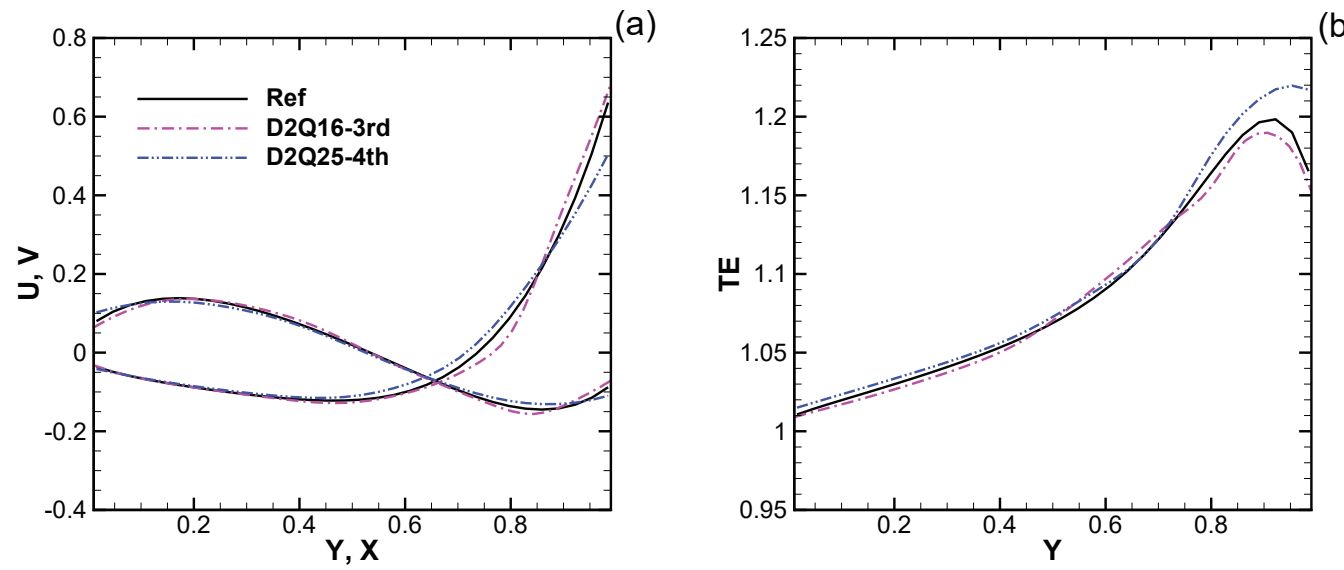

(b)

FIGURE 6. (a) Vertical and horizontal velocity profiles along the centerlines of the cavity and (b) temperature along the vertical centerline of the cavity for $M a=1$ and $K n=0.1$.

the same velocity profile is expected to be obtained for the continuum flow. It can be seen from Figs. 4(a) and 4(b) that for the given discrete velocity sets, the velocity profiles across the centerlines of cavity agree well with the reference solutions when $K n=0.001$ and 0.01 . However, things are different when the rarefaction effect plays an important role. When $K n=0.1$ in the transition regime, D2Q37 with both third and fourth order approximations shows same results, which are overall in reasonable agreement with the reference solutions, except that near the moving lid where it is about 15\% larger than the reference solution. In addition, it is interesting to see that D2Q16 with third order approximation give the best prediction of velocity distributions near the wall boundaries, where the deviation of the present result from the reference solution is less than $4 \%$. The result from D2Q17 with third order approximation is worst when compared with those from the rest of velocity sets.

The temperature distributions along the vertical centerline of the cavity for $K n=0.001,0.01$, and 0.1 are plotted in Fig. 5. Expectedly, for D2Q37, the results from fourth order truncation is much better than those from the third one, due to the missing term in the heat flux for the third approximation [7]. However, it is surprising to see that with the third order approximation of the VDF, the results of D2Q16 is better than those of D2Q37 and D2Q17. In particular, for $K n=0.1$, the result of $\mathbf{D} 2 \mathbf{Q} 16$ with the third truncation of VDF is even better than that of the $\mathbf{D} 2 \mathbf{Q} 37$ with the fourth order approximation. In other words, the discrete velocity sets with even number work much better than the odd one which has a zero molecular velocity. This finding is consistent with those from Meng et al [9] and Chikatamarla et al [12].

Finally, we also compare the temperature and velocity obtained from two off-lattice Gauss-Hermite discrete velocity sets, i.e., D2Q16 and D2Q25 in Fig. 6. The third and fourth order approximations are used for D2Q16 and 
D2Q25, respectively, in case that the corresponding moments can be accurately obtained. The same as the above observation, D2Q16 gives much better results than the D2Q25, particularly, near the wall boundaries.

\section{Conclusions}

In this work, a high order discrete unified gas kinetic scheme has been proposed for high speed flow on the basis of the high order truncation of the Hermite polynomial. The performances of two compact on-lattice velocity sets, i.e. D2Q17 and D2Q37, as well as the Gauss-Hermite tensor product off-lattice velocity sets, i.e. D2Q16 and D2Q25, associated with the third and fourth order of Hermite polynomial expansion of the VDF, are assessed by simulating the Sod shock tube flow and two-dimensional lid driven cavity flow. It is confirmed from the simulation results that the third and fourth order Hermite polynomial expansions could accurately reproduce the isothermal, and thermal and fully compressible flow, respectively. In addition, it is expected to see that the performance of $\mathbf{D} 2 \mathbf{Q} 17$ is worse than those from D2Q37 and D2Q25. However, it is surprisingly found that the performance of D2Q16 is better than that of $\mathbf{D} 2 \mathbf{Q} 17$, and it is even better than that of $\mathbf{D} 2 \mathbf{Q} 37$ and $\mathbf{D} 2 \mathbf{Q} 25$ for flows in the early transition regime, suggesting that the even number of velocity points without the zero velocity show a better capacity than the odd one in predicting the rarefaction effect.

In summary, it is possible using the fourth high order Hermite polynomial expansion of the equilibrium distribution function associated with the compact discrete velocity sets of D2Q37 and D2Q25 to reproduce the thermal and fully compressible flow, but the available compact discrete velocity sets are not sufficient to produce the rarefied effect, it requires more velocity points to capture the nonequilibrium phenomena. In addition, the molecular velocity space discretized without the zero velocity may be a promising way to reduce the number of the velocity point.

\section{ACKNOWLEDGMENTS}

This work is financially supported by the UK's Engineering and Physical Sciences Research Council (EPSRC) under grants EP/M021475/1.

\section{REFERENCES}

[1] S. Chapman, T. G. Cowling, and D. Burnett, The mathematical theory of non-uniform gases: an account of the kinetic theory of viscosity, thermal conduction and diffusion in gases (Cambridge university press, 1990).

[2] Z. Guo and C. Shu, Lattice Boltzmann method and its applications in engineering, Vol. 3 (World Scientific, 2013).

[3] K. Xu, Journal of Computational Physics 171, 289-335 (2001).

[4] J. Yang and J. Huang, Journal of Computational Physics 120, 323-339 (1995).

[5] P. Wang, M. T. Ho, L. Wu, Z. Guo, and Y. Zhang, Computers \& Fluids 161, 33-46 (2018).

[6] X. Shan, X.-F. Yuan, and H. Chen, Journal of Fluid Mechanics 550, 413-441 (2006).

[7] C. Coreixas, G. Wissocq, G. Puigt, J.-F. Boussuge, and P. Sagaut, Physical Review E 96, p. 033306 (2017).

[8] J. Meng and Y. Zhang, Journal of Computational Physics 230, 835-849 (2011).

[9] J. Meng and Y. Zhang, Physical Review E 83, p. 036704 (2011).

[10] P. C. Philippi, L. A. Hegele Jr, L. O. Dos Santos, and R. Surmas, Physical Review E 73, p. 056702 (2006).

[11] H. Grad, Communications on pure and applied mathematics 2, 331-407 (1949).

[12] S. S. Chikatamarla and I. V. Karlin, Physical review letters 97, p. 190601 (2006).

[13] Z. Guo, K. Xu, and R. Wang, Physical Review E 88, p. 033305 (2013).

[14] Z. Guo, R. Wang, and K. Xu, Physical Review E 91, p. 033313 (2015).

[15] P. L. Bhatnagar, E. P. Gross, and M. Krook, Physical Review 94, p. 511 (1954).

[16] X. Shan, Journal of Computational Science 17, 475-481 (2016).

[17] P. Wang, L. Zhu, Z. Guo, and K. Xu, Communications in Computational Physics 17, 657-681 (2015).

[18] E. Shakhov, Fluid Dynamics 3, 95-96 (1968).

[19] L. H. Holway Jr, The physics of fluids 9, 1658-1673 (1966).

[20] S. S. Chikatamarla and I. V. Karlin, Physical Review E 79, p. 046701 (2009).

[21] P. Wang, L.-P. Wang, and Z. Guo, Physical Review E 94, p. 043304 (2016). 